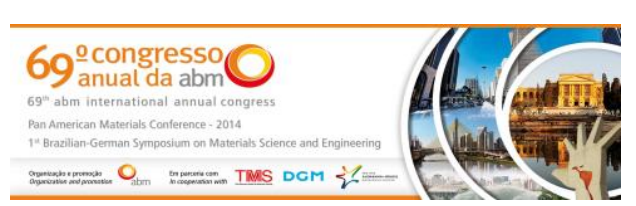

Tema: Gestão de meio ambiente e recuperação e tratamento de rejeitos

\title{
CINÉTICA DA REDUÇÃO DE FERRITA DE ZINCO POR MISTURAS $\mathrm{CO}-\mathrm{CO}_{2}{ }^{*}$
}

\section{Resumo}

\author{
Mery Cecilia Gómez Marroquin ${ }^{1}$ \\ Jose Carlos D'Abreu² \\ Helio Marques Kohler ${ }^{3}$ \\ Edelink Efrain Tinoco Falero ${ }^{4}$
}

Um importante problema das indústrias de fabricação de aço, decorrentes do uso de sucata de aço, é o de recuperação dos metais contidos nos pós e lamas produzidos nos fornos elétricos a arco (FEA) e nos conversores LD/FOB. Cerca de 10 a $20 \mathrm{~kg}$ de pó por tonelada de aço são gerados nos Fornos Elétricos a Arco, considerados resíduos perigosos pelos órgãos fiscalizadores (classe I - material perigoso/NBR). Vários processos, utilizando tecnologias distintas (pirometalúrgicas e hidrometalúrgicas), buscam a recuperação técnico-econômica principalmente do $\mathrm{Fe}$ e do Zn, contidos nesses resíduos. Neste contexto, estudos básicos sobre a redução carbotérmica dos constituintes dos pós de aciaria, permanecem sob estudo. 0 presente trabalho foca a redução de ferrita de zinco gerada em laboratório, principal composto de zinco presente nos pós de aciaria, por meio de $\mathrm{CO}$ puro e misturas $\mathrm{CO}-\mathrm{CO}_{2}$. A fenomenologia da redução considera a decomposição prévia seguida de reduções simultâneas, bem como as significativas influencias da temperatura, do tempo e do teor de CO.

Palavras-chave: Reciclagem; Pós de aciaria; Cinética; Ferrita de zinco.

\section{Abstract}

\section{REDUCTION OF ZINC FERRITE BY CO-CO 2 MIXTURES}

An important problem of steelmaking, arising from the use of scraps in steel industries are the recovery of metals contained in the dust and sludge produced in electric arc furnaces (EAF) and LD converters / FOB. About 10 to $20 \mathrm{~kg}$ of dust per ton of steel are generated in EAF, which are considered hazardous wastes by the environmental controlling agencies (Class I - hazardous materials / NBR). Several processes using different technologies (pyrometallurgical and hydrometallurgical), has been used in trying to recover mainly $\mathrm{Fe}$ and $\mathrm{Zn}$ contained in these wastes, focusing on both technical and economic aspects. In this context, theoretical and phenomenological researches are being conducted. The present study focuses the reduction of zinc ferrite produced in laboratory, using pure $\mathrm{CO}$ and $\mathrm{CO}-\mathrm{CO}_{2}$ mixtures. The phenomenology is supported by a prior decomposition of zinc ferrite followed by simultaneous reductions, and the significant effecs of the temperature, time and $\%$ CO.

Keywords: Recycling; Steel making dust; Kinetics; Zinc ferrite.

1 Eng. Metalúrgico, DSc, Professor do departamento de Metalurgia (FIGMM), Universidad Nacional de Ingenieria (UNI), Lima, Perú.

2 Eng. Metalúrgico, DSc, Professor Emérito do Departamento de Ciências dos Materiais e Metalurgia (DEMa), Pontifícia Universidade Católica do Rio de Janeiro (PUC-Rio), RJ, Brasil.

3 Eng. Metalúrgico, PhD, Professor do departamento de Mecânica da universidade estadual de Rio de Janeiro (UERJ), RJ, Brasil.

4 Eng. Metalúrgico, MSc, Doutorando em Engenharia Metalúrgica e Materiais, Depto. de Ciências dos Materiais e Metalurgia (DEMa), PUC-Rio, RJ, Brasil.

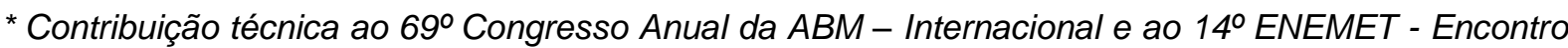
Nacional de Estudantes de Engenharia Metalúrgica, de Materiais e de Minas,21 a 25 de julho de 2014, São Paulo, SP, Brasil.
} 


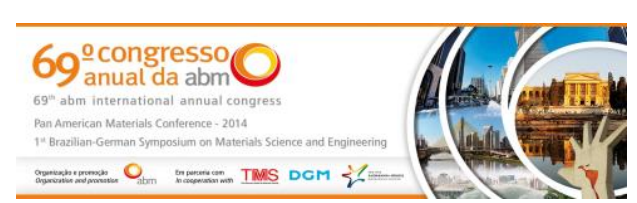

\section{INTRODUÇÃO}

O zinco presente nos pós de aciaria elétrica pode chegar a 30\% na sua composição, dos quais em torno de $70 \%$ está na forma de ferrita de zinco e o restante principalmente como óxido de zinco e zinco metálico [1]. Portanto, alem da ferrita de zinco constituir uma realidade importante, acresce o fato de que a redução deste composto ainda não está completamente estudada e que sua quantidade aumenta nas poeiras de aciaria, à medida que é incrementada a reciclagem de sucata de aço galvanizada.

Segundo Jyh-Jen [2], a redução carbotérmica da ferrita de zinco em temperaturas entre 1073 e $1473 \mathrm{~K}$, apresenta uma energia de ativação aparente de 92,91 kJ/mol.

A fenomenologia de redução proposta considera que este composto $\left(\mathrm{ZnFe}_{2} \mathrm{O}_{4}\right)$ primeiramente se decompõe em $\mathrm{ZnO}$ e $\mathrm{Fe}_{2} \mathrm{O}_{3}$ e somente após ocorre a redução dos mesmos, conduzindo ao incremento simultaneo da formação de poros e o aumento da superfície específica. O incremento do volume do sólido é observado durante a redução dos óxidos de ferro e, mais para o fim da redução, também uma contração, devida a sinterização do ferro e ao fechamento dos poros.

Visando contribuir para um maior entendimento da fenomenologia de redução deste composto, o presente trabalho é parte de um projeto que tem como objetivo geral analisar o comportamento cinético e morfológico da redução da ferrita de zinco contida nas poeiras de aciaria, por misturas $\mathrm{CO}$ e $\mathrm{CO}_{2}$.

\section{MATERIAIS E MÉTODOS}

Como matéria prima para este trabalho foi utilizada a ferrita de zinco produzida em laboratório, a partir da mistura básica $\mathrm{Fe} 2 \mathrm{O} 3 / \mathrm{ZnO}: 1 / 1$, segundo técnica desenvolvida pelos pesquisadores Bid e Pradhan [3]; Gómez e D’Abreu [4,5], e posteriormente aglomeradas na forma de briquetes cilíndricos [6].

A ferrita de zinco produzida foi caracterizada estruturalmente via Difração de Raios $X$. Para isto, usou-se o difratómetro SIEMENS, modelo D5000, I=30 A, V=40 kV com ânodo de cobre $\left(\lambda=1,5406 \mathrm{~A}^{\circ}\right.$, CuK- $\left.\alpha\right)$ e o software DiffraccPlus, Topas versão 2.1 da Bruker AXS de análise quantitativa, pelo método de Rietveld [7].

Em seguida amostras de ferrita de zinco foram caracterizadas morfologicamente via Microscópio Eletrônico de Varredura-MEV (DSM 960 Zeiss West Germany V=20kV) e Microscópio Eletrônico de Transmissão-MET (Modelo JEOL 2010 V=200kV).

A caracterização física da ferrita de zinco foi realizada usando os seguintes equipamentos:

- Multipycnometer quanta chrome $\mathrm{V}=120 \mathrm{~V}$ e $\mathrm{P}=18 \mathrm{psi}$, para a determinação da massa especifica.

- Mastersizer $\mu$ Versão 2.12 da Malvern Instruments, para a determinação da distribuição do tamanho e superfície especifica.

Em seguida, amostras pesando aproximadamente $7 \mathrm{~g}$ foram aglomeradas na forma de briquetes cilíndricos, com 2,54 cm de diâmetro.

Finalmente, testes de redução dos briquetes foram realizados num forno elétrico tubular acoplado a uma linha de gases de $\mathrm{CO}, \mathrm{CO}_{2}$ e N2, nas temperaturas de 1073, 1173,1273 e $1373 \mathrm{~K}$. Os experimentos utilizaram tempos de 8, 56,5 e 105 minutos, e composições gasosas de $100 \%$ de $\mathrm{CO}$ e $50 \mathrm{CO} \%-50 \% \mathrm{CO}_{2}$.

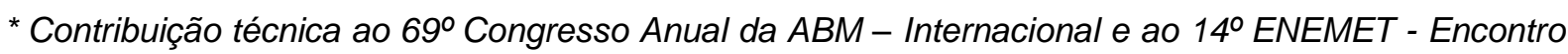
Nacional de Estudantes de Engenharia Metalúrgica, de Materiais e de Minas,21 a 25 de julho de 2014, São Paulo, SP, Brasil.
} 


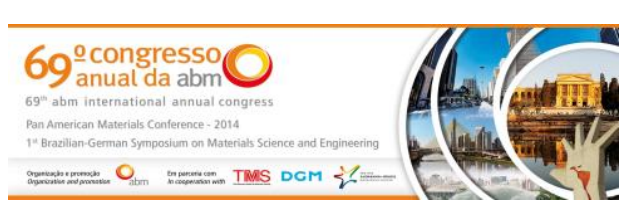

\section{RESULTADOS E DISCUSSÕES}

\subsection{Caracterização Estrutural}

$\mathrm{Na}$ Figura1 é apresentado o difratograma da ferrita de zinco produzida em laboratório. Analise realizada pelo método Rietveld, mostrou as seguintes percentagens para seus constituintes: ferrita de zinco $\left(94,82 \% \mathrm{ZnFe}_{2} \mathrm{O}_{4}\right)$, óxido de ferro III $\left(3,48 \% \mathrm{Fe}_{2} \mathrm{O}_{3}\right)$ e óxido de zinco $(1,70 \% \mathrm{ZnO})$.

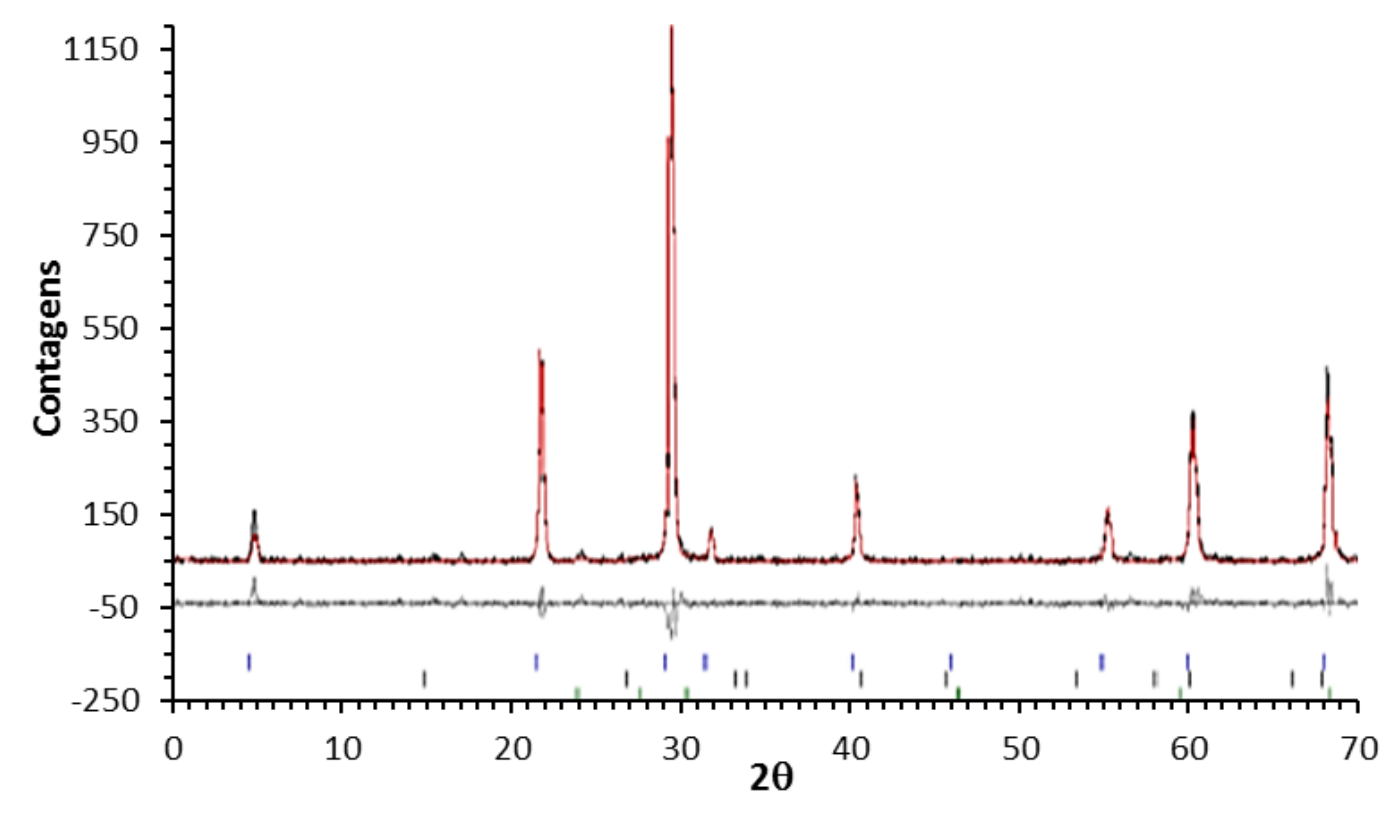

Figura 1 - Difratograma da ferrita de zinco utilizada no método de Rietveld

O método de Rietveld mostrou ser uma ferramenta importante não apenas para a determinação do teor de ferrita de zinco, mas principalmente para 0 acompanhamento de sua redução e determinação das outras fases de interesse presentes nas amostras de ferrita de zinco, como o $\mathrm{Fe}_{2} \mathrm{O}_{3}$ e o $\mathrm{ZnO}$.

\subsection{Caracterização microscópica}

Para complementar o trabalho de identificação e quantificação das fases nas amostras de ferrita de zinco, usou-se as Microscopias Eletrônicas de VarreduraMEV e de Transmissão-MET.

$\mathrm{Na}$ Figura 2, a imagem de $\mathrm{MEV}$ da amostra de $\mathrm{ZnFe}_{2} \mathrm{O}_{4}$ na condição como produzida, exibe a presença majoritária de ferrita de zinco na forma de glóbulos brancos, além de uma grande presença de poros, morfologias de grande importância para o comportamento cinético da redução deste composto.

\footnotetext{
* Contribuição técnica ao $69^{\circ}$ Congresso Anual da ABM - Internacional e ao 14ํㅡㄹ ENEMET - Encontro Nacional de Estudantes de Engenharia Metalúrgica, de Materiais e de Minas,21 a 25 de julho de 2014, São Paulo, SP, Brasil.
} 

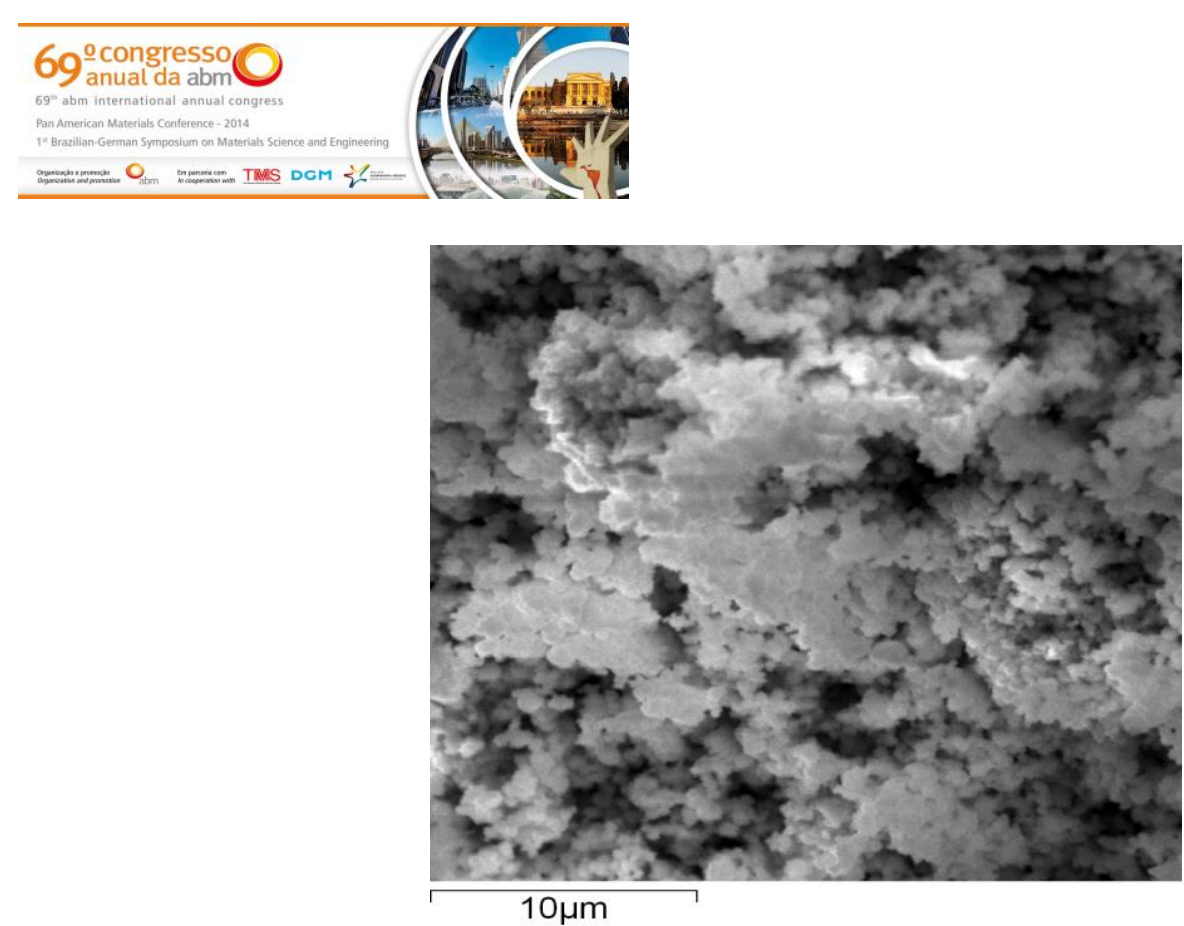

Figure 2 - Imagem no MEV da amostra de ferrita de Zinco

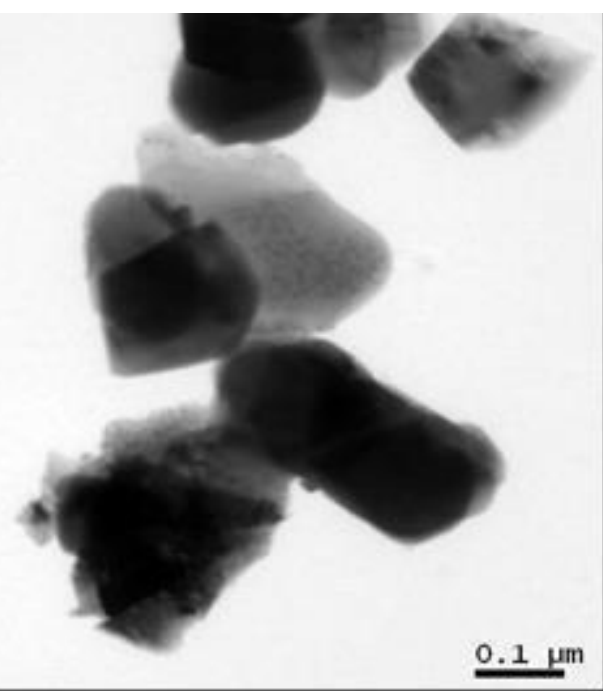

Figure 3 - Imagem no MET da amostra de ferrita de zinco equimolar, nanométrica

Na imagem de MET da Figura 3, foi possível observar que as partículas de ferrita de zinco apresentaram tamanhos na faixa de 100 a $200 \mathrm{~nm}$ e morfologias facetadas e arredondadas, algumas delas aglutinadas ou superpostas, características importantes a serem consideradas no seu desempenho sob redução.

\subsection{Caracterização Física}

Foram determinados o tamanho médio das partículas, sua superfície especifica, massa especifica e, para os briquetes produzidos após prensagem, sua porosidade. A seguir são apresentados os valores obtidos:

- Diâmetro médio das partículas: $37 \mu \mathrm{m}$

- Superfície específica: 0,0067 $\mathrm{m}^{2} / \mathrm{g}$

- Massa especifica: $5,42 \mathrm{~g} / \mathrm{cm}^{3}$

- Porosidade do briquete da amostra: 0,45

Com base nesses resultados foi definida a mínima carga de briquetagem para a aglomeração das amostras de ferrita de zinco, que promovesse a menor influencia na variação da porosidade e na sua uniformidade, no aglomerado. Após uma série

* Contribuição técnica ao 69 Congresso Anual da ABM - Internacional e ao 14ํㅡㄹ ENEMET - Encontro Nacional de Estudantes de Engenharia Metalúrgica, de Materiais e de Minas,21 a 25 de julho de 2014, São Paulo, SP, Brasil. 


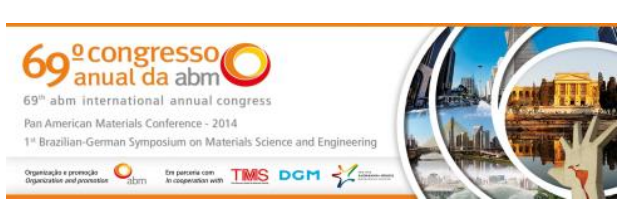

de testes de briquetagem, foi escolhido o valor de 2000kgf para a carga de compressão. Na Figura 4 é mostrada uma ilustração do briquete de 7 gramas usado nos experimentos de redução.

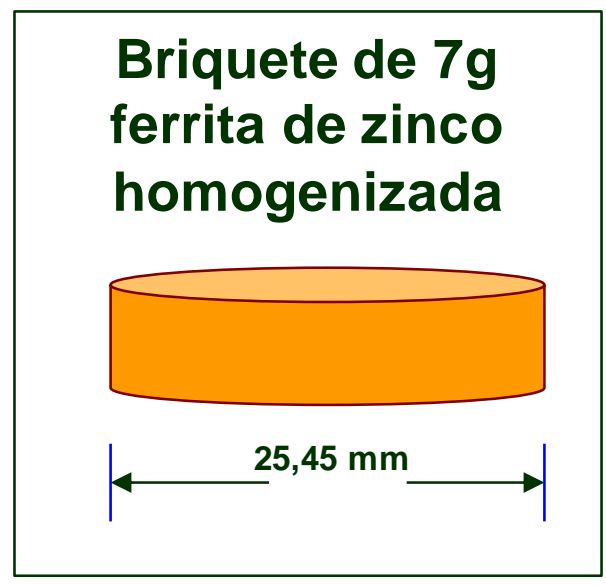

Figura 4 - Briquete Utilizado nas Experiencias de Redução

\subsection{Redução}

As temperaturas e composições de $\mathrm{CO}-\mathrm{CO}_{2}$ para os testes de redução foram escolhidas a partir do Diagrama Operacional de Predominância de Fases (DOPF) para o sistema Zn-Fe-C-O, elaborado pelos autores [8].

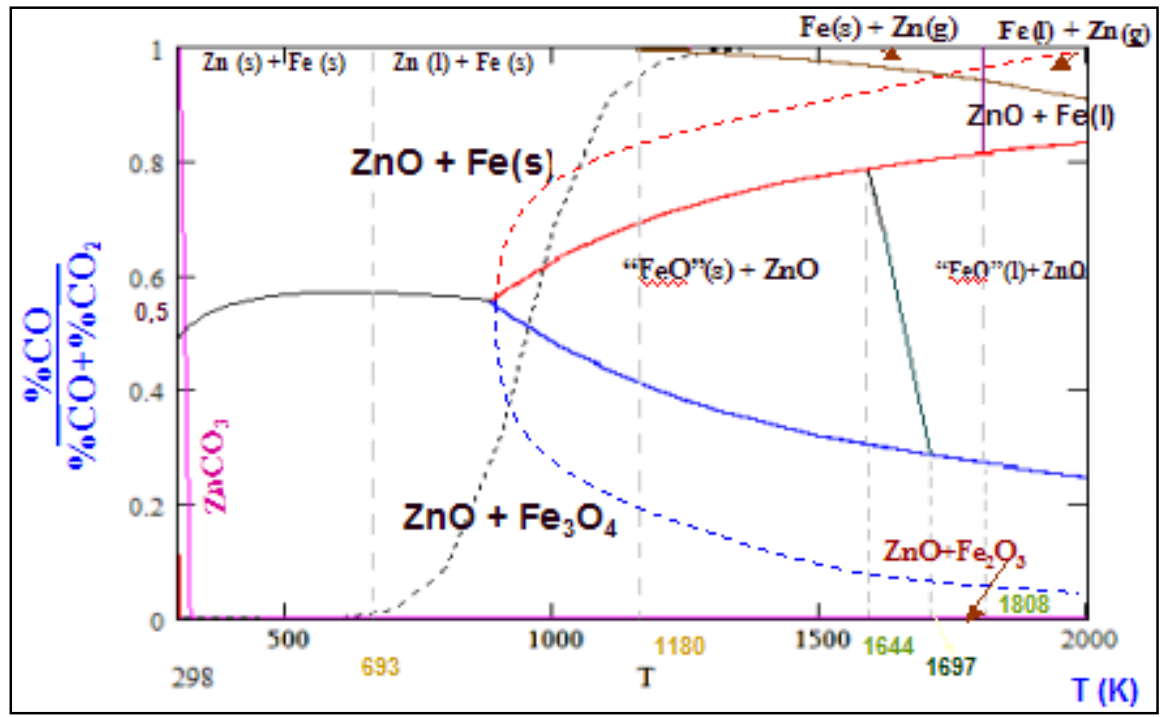

Figura 5 - Diagrama operacional de predominância de fases do sistema Zn-Fe-C-O

A Figura 5 apresenta o DOPF para o sistema $\mathrm{Zn}-\mathrm{Fe}-\mathrm{C}-\mathrm{O}$, assinalando os equilíbrios $\mathrm{ZnO}-\mathrm{Zn}$ e $\mathrm{Fe}_{2} \mathrm{O}_{3}-\mathrm{Fe}_{3} \mathrm{O}_{4}-\mathrm{FeO}-\mathrm{Fe}$, sob atmosferas de $\mathrm{CO}-\mathrm{CO}_{2}$. Os campos de predominância das várias espécies também são apresentados. A seguir estão propostas as reações de redução da ferrita de zinco pelo CO (1) e via intermediários gasosos (3):

$$
\begin{aligned}
& \mathrm{ZnO} \cdot \mathrm{Fe}_{2} \mathrm{O}_{3(\mathrm{~s})}+4 \mathrm{CO}_{(\mathrm{g})}=\mathrm{Zn}(\mathrm{g} / \mathrm{l})+2 \mathrm{Fe}_{(\mathrm{s} / \mathrm{l})}+4 \mathrm{CO}_{2(\mathrm{~g})} \\
& \mathrm{CO}_{2(\mathrm{~g})}+\mathrm{C}_{(\mathrm{s})}=2 \mathrm{CO}_{(\mathrm{g})}
\end{aligned}
$$

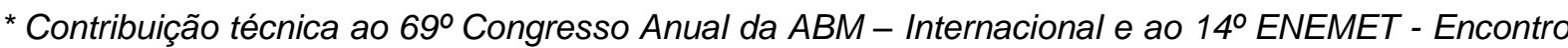
Nacional de Estudantes de Engenharia Metalúrgica, de Materiais e de Minas,21 a 25 de julho de 2014, São Paulo, SP, Brasil.
} 


$$
\mathrm{ZnO} \cdot \mathrm{Fe}_{2} \mathrm{O}_{3(\mathrm{~s})}+4 \mathrm{C}=\mathrm{Zn}(\mathrm{g} /)+2 \mathrm{Fe}_{(\mathrm{s} / l)}+4 \mathrm{CO}
$$

Para a realização dos experimentos os parâmetros de redução da ferrita de zinco foram escolhidos com base no DOPF, considerando duas condições: redução completa e redução parcial. Os valores correspondentes foram:

a- Composições gasosas: $100 \% \mathrm{CO}$ e mistura $50 \% \mathrm{CO}-50 \% \mathrm{CO}_{2}$

b- Temperaturas: $800,900,1000$ e $1100^{\circ} \mathrm{C}$

Nas Figuras 6 e 7 estão apresentadas as curvas de redução dos briquetes de ferrita de zinco (7 gramas), para a mistura 50\% CO $-50 \% \mathrm{CO}_{2}$ e $100 \% \mathrm{CO}$, respectivamente.

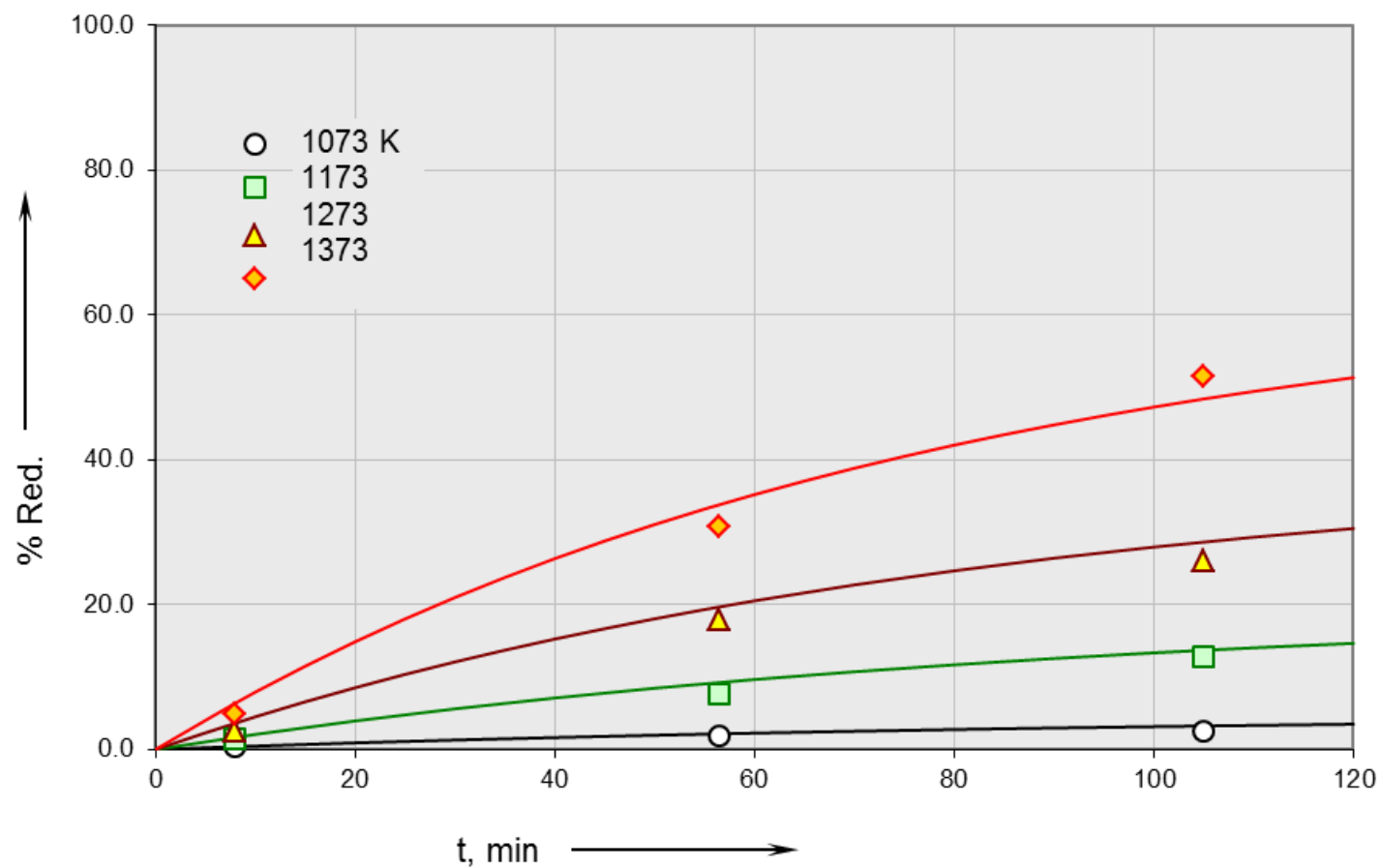

Figure 6 - Reduções da ferrita de zinco por mistura gasosa 50\% CO - 50\% $\mathrm{CO}_{2}$

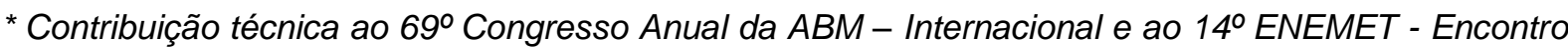
Nacional de Estudantes de Engenharia Metalúrgica, de Materiais e de Minas,21 a 25 de julho de 2014, São Paulo, SP, Brasil. 


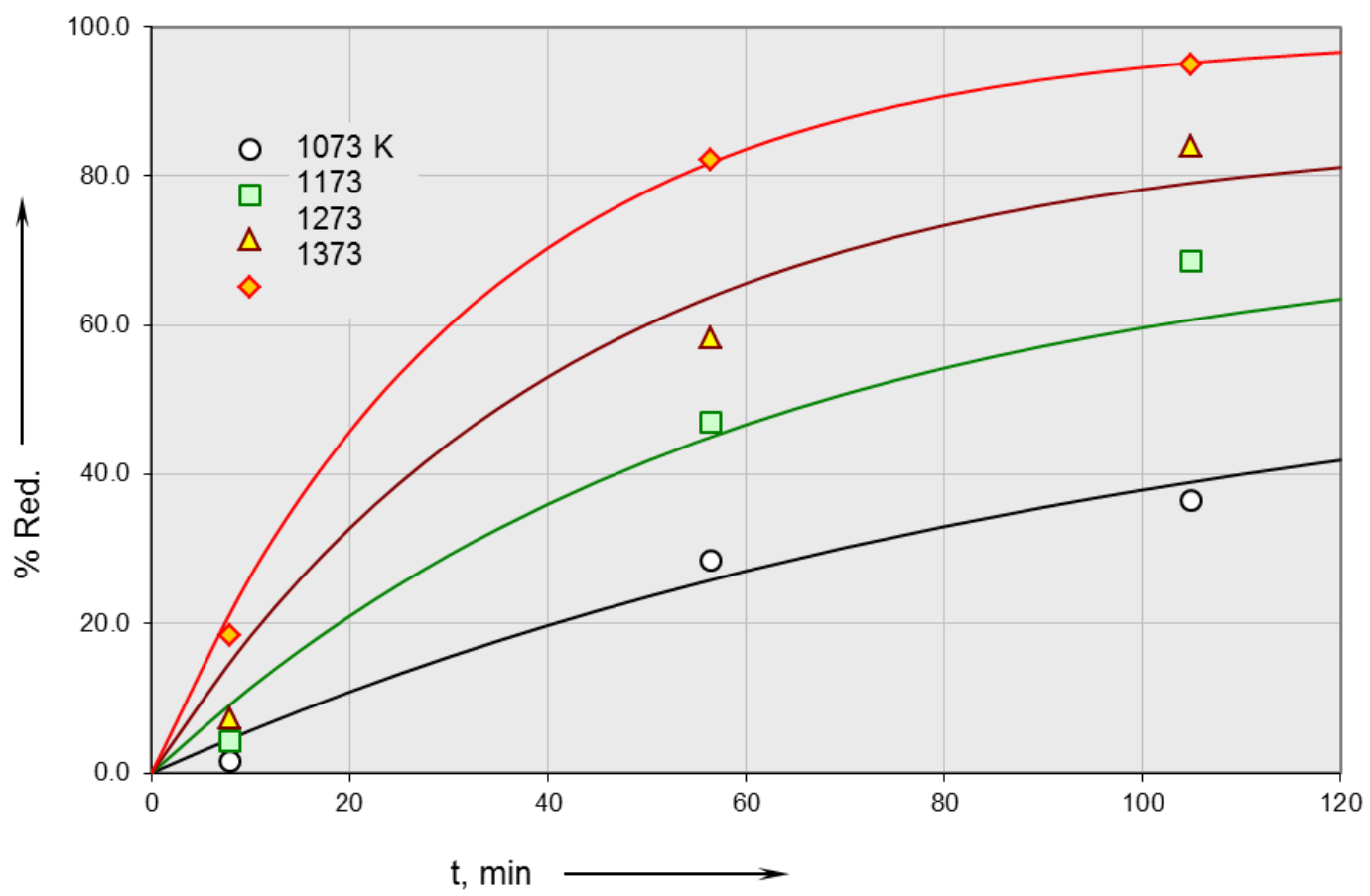

Figure 7 - Reduções da ferrita de zinco por 100\% CO

As reduções, tanto a realizada com $100 \%$ de $\mathrm{CO}$ como com $50 \%$ de CO, mostraram ser significativamente dependentes da temperatura, estando de acordo com as cinéticas de redução dos compostos $\mathrm{ZnO}$ e $\mathrm{Fe}_{2} \mathrm{O}_{3}$, isoladamente. Por outro lado, os valores obtidos também confirmaram que o mecanismo de redução da ferrita de zinco se processa via as reações simultâneas dos dois compostos, $\mathrm{ZnO}$ e $\mathrm{Fe}_{2} \mathrm{O}$, atendidas as priorizações termodinâmicas estabelecidas no DOPF. Outro fato importante a considerar, diz respeito às fases metálicas obtidas nas reduções isotérmicas, pois, dependendo das condições experimentais de temperatura, elas foram geradas em diferentes estados físicos (Zinco: liquido e gasoso; Ferro: sólido). Tais fatos, além de confirmarem a proposta de modelo cinético previamente apresentada pelos autores [8], também mostrou ser determinante na analise dos fatores que conduziram a conversões maiores e a dR/dt mais altos (Figuras 6 e 7).

\section{CONCLUSÕES}

Os resultados obtidos permitiram concluir:

1) O diagrama operacional de predominância de fases (DOPF) para o sistema $\mathrm{Zn}-\mathrm{Fe}-\mathrm{C}-\mathrm{O}$, forneceu informações fundamentais para os experimentos cinéticos, principalmente as variáveis temperatura, \% CO e espécies geradas;

2) As caracterizações microscópicas das amostras de ferrita de zinco em MEV e no MET permitiram observar a presença de grupos de partículas (clusters), formados por partículas individualizadas, com tamanhos entre 100 e 200nm;

3) Os aumentos da temperatura e do tempo acarretaram incrementos significativos na redução da ferrita de zinco, bem como distintos estados físicos da fase metálica produzida;

5) O aumento da percentagem de $\mathrm{CO}$ na mistura gasosa promoveu velocidades de redução mais altas, bem como maiores conversões finais.

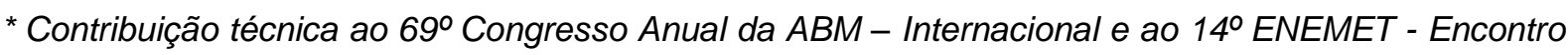
Nacional de Estudantes de Engenharia Metalúrgica, de Materiais e de Minas,21 a 25 de julho de 2014, São Paulo, SP, Brasil.
} 


\section{Agradecimentos}

À Capes e ao CNPq pela bolsa de pós-graduação. A Raimundo Nonato Rodrigues Filho, pela ajuda nas analises instrumentais.

\section{REFERÊNCIAS}

1 Hsi-Kuei C, Ching-Yi Y. A study on the preparation of zinc ferrite. Scandinavian Journal of Metallurgy. 2001; 30.

2 Jyh -Jen L, Chun- I L, Hsi-Kuei C. Carbothermal reduction of zinc ferrite. Metallurgical and Materials Transactions.2001; 32B: 1033-1040.

3 Bid S, Pradhan Sk. Preparation of zinc ferrite by high-energy ball-milling and microestruture characterization by Reitveld's analysis. Materials Chemistry and Physics, 2003;82: 27-37.

4 Gómez MMC, D’Abreu JC, Kohler MH. Contribuição ao estudo da formação da ferrita de zinco contida nas poeiras de aciaria elétrica. Trabalho apresentado no II Seminário de Auto-Redução e Aglomeração a Frio, do $59^{\circ}$ Congresso Anual da ABM Internacional, São Paulo, 2004.

5 Gómez MMC, D’Abreu JC, Kohler MH. Estudo cinético da formação de ferrita de zinco. Trabalho a ser apresentado no tema matérias primas para área de redução carvão, minério de ferro, fundentes e adições do XXXVI Seminário de Redução de Minério de Ferro e Matérias Primas. Minas Gerais, 2006.

6 Özbayoğlu G, Hiçyilmaz C, Akdemir Ü. Briqueting of zinc oxide fines. Powder Technology.1993; 77: 153-158.

7 Tong FL. Reduction mechanisms and behaviour of zinc ferrite-Part 1: pure Zn Fe2O4. Received by the Institution of Mining and Metallurgy; Paper published in Trans. Instn Min. Metall. (Sect. C: Mineral Processes. Extractive Metallurgy); 2001.

8 Gómez MMC, D’Abreu JC. Estudo termodinâmico da redução da ferrita de zinco. Trabalho apresentado no III Seminário de Auto-Redução e Aglomeração a Frio, do $60^{\circ}$ Congresso Anual da ABM Internacional, Belo Horizonte, 2005.

\footnotetext{
* Contribuição técnica ao $69^{\circ}$ Congresso Anual da ABM - Internacional e ao 14ํㅡㄹ ENEMET - Encontro Nacional de Estudantes de Engenharia Metalúrgica, de Materiais e de Minas,21 a 25 de julho de 2014, São Paulo, SP, Brasil.
} 